\title{
The web-based multiplex PCR primer design software Ultiplex and the associated experimental workflow: up to 100- plex multiplicity
}

Jie Yuan ${ }^{1,2+}, \mathrm{Ji}_{\mathrm{Yi}^{3+}}$, Meixiao Zhan ${ }^{4}$, Qingqing Xie ${ }^{3}$, Ting Ting Zhen ${ }^{3}$, Jian Zhou ${ }^{3}$, Zeqing $\mathrm{Li}^{3,5^{*+}}$ and Zhou Li ${ }^{1 *+}$ (D)

\begin{abstract}
Background: A large number of variants have been employed in various medical applications, such as providing medication instructions, disease susceptibility testing, paternity testing, and tumour diagnosis. A high multiplicity PCR will outperform other technologies because of its lower cost, reaction time and sample consumption. To conduct a multiplex PCR with higher than 100 plex multiplicity, primers need to be carefully designed to avoid the formation of secondary structures and nonspecific amplification between primers, templates and products. Thus, a user-friendly, highly automated and highly user-defined web-based multiplex PCR primer design software is needed to minimize the work of primer design and experimental verification.
\end{abstract}

Results: Ultiplex was developed as a free online multiplex primer design tool with a user-friendly web-based interface (http://ultiplex.igenebook.cn). To evaluate the performance of Ultiplex, 294 out of 295 (99.7\%) target primers were successfully designed. A total of 275 targets produced qualified primers after primer filtration, and 271 of those targets were successfully clustered into one compatible PCR group and could be covered by 108 primers. The designed primer group stably detected the rs 28934573 ( $C>$ T) mutation at lower than a $0.25 \%$ mutation rate in a series of samples with different ratios of HCT-15 and HaCaT cell line DNA.

Conclusion: Ultiplex is a web-based multiplex PCR primer tool that has several functions, including batch design and compatibility checking for the exclusion of mutual secondary structures and mutual false alignments across the whole genome. It offers flexible arguments for users to define their own references, primer Tm values, product lengths, plex numbers and tag oligos. With its user-friendly reports and web-based interface, Ultiplex will provide assistance for biological applications and research involving genomic variants.

Keywords: Primer design, Multiplex, Software, Web interface, Variant

\footnotetext{
*Correspondence: 110655575@qq.com; gzlizhou@smu.edu.cn

†Jie Yuan and Ji Yi contributed equally to this work.

†Zeqing Li and Zhou Li contributed equally to this work.

${ }^{3}$ Medical Department, Wuhan Igenebook Biotechnology Co., Ltd, Floor 3,

building 1, Zone B, Gaonong Biological Park, 888 Gaoxin Avenue, Wuhan

430014, Hubei Province, China

${ }^{1}$ General Surgery Center, Zhujiang Hospital, Southern Medical University, 253

Industrial Boulevard, Guangzhou 510280, Guangdong Province, China

Full list of author information is available at the end of the article
}

(c) The Author(s). 2021 Open Access This article is licensed under a Creative Commons Attribution 4.0 International License, which permits use, sharing, adaptation, distribution and reproduction in any medium or format, as long as you give appropriate credit to the original author(s) and the source, provide a link to the Creative Commons licence, and indicate if changes were made. The images or other third party material in this article are included in the article's Creative Commons licence, unless indicated otherwise in a credit line to the material. If material is not included in the article's Creative Commons licence and your intended use is not permitted by statutory regulation or exceeds the permitted use, you will need to obtain permission directly from the copyright holder. To view a copy of this licence, visit http://creativecommons.org/licenses/by/4.0/. The Creative Commons Public Domain Dedication waiver (http://creativecommons.org/publicdomain/zero/1.0/) applies to the data made available in this article, unless otherwise stated in a credit line to the data. 


\section{Background}

The relationship between phenotype and variations in the human genome has been progressively clarified. Over 88 million variants (84.7 million single-nucleotide polymorphisms (SNPs), 3.6 million short insertions/deletions (indels), and 60,000 structural variants) had been characterized as of 2015 [1]. The Online Mendelian Inheritance in Man $\left(\mathrm{OMIM}^{\mathrm{sM}}\right)$ database had 13,005 entries on October 1, 2001 [2]. A large number of variants have been employed in various applications related to people's lives, such as providing medication instructions, disease susceptibility testing, paternity testing, and tumour diagnosis. Hereditary nonpolyposis colorectal cancer syndrome (HNPCC) is the most common hereditary form of colorectal cancer. Patients with HNPCC exhibit an up to $80 \%$ increase in the lifetime risk of colorectal cancer and an up to $60 \%$ increase in the lifetime risk of endometrial cancer [3]. HNPCC results from a germline mutation in one of four mismatch repair $(M M R)$ genes [4]. MMR genes and other hereditary tumour genes also contribute to primary hepatocellular carcinoma, breast cancer and other cancers [5]. Early detection of these hereditary gene mutations provides adequate time for tumour prevention and periodic examinations such as colonoscopy.

To detect hundreds of genome variants (such as hereditary tumour gene mutations), whole-genome sequencing and target capture sequencing are usually needed to cover such a large number of variants [6]. However, once the target number of multiplex PCRs is within the range of hundreds, PCR-based target sequencing will outperform these other technologies due to its lower cost, reaction time and sample consumption. For example, whole exon sequencing (WES), one kind of target capture sequencing method, which can cover 30-60 million bases of the human genome, usually costs $\$ 150 \sim 300$ per sample in China, needs a $200 \mathrm{ng} \sim 2 \mu \mathrm{g}$ DNA sample and takes $2-3$ days to complete library construction. Another method suitable for genome variant detection is HumanCytoSNP12 chips (Illumina), which cover $\sim 301,000$ SNPs and other genetic markers. However, HumanCytoSNP-12 chips still cost $\$ 267$ per sample in China, need a $200 \mathrm{ng}$ DNA sample and take 3 days to complete. When interrogating less than 200 genomic variants, self-tailored multiplex PCR will be more suitable, economical and efficient. The cost of self-tailored multiplex PCR only relies on primer synthesis, PCR reagents, and sequencing, which usually costs approximately $\$ 15 \sim 30$ in China. In addition, DNA sample consumption during multiplex PCR is less than $100 \mathrm{ng}$, and the library construction only takes half a day to complete.

Self-tailored multiplex PCR methods mainly depend on well-designed primers. However, open-source multiplex PCR primer design software is rarely applicable (Table 1), particularly at the 100-plex level. To conduct multiplex PCR, primers need to be carefully designed to avoid the formation of secondary structures and nonspecific amplification between primers, templates and products [7]. Primer3 is the most popular open-source group of programs, programming libraries and web interfaces for assisting researchers with PCR primer design [8]. However, these programs lack multiplex primer clustering and filtering functions. Primer-BLAST allows users to design new target-specific primers in a single step and to check the specificity of pre-existing primers [9]. However, it is only suitable for designing one primer pair at a time based on web interfaces. Other primer design software programs, such as Oligo 7 [10], PrimerSelect [11], Primer Premier [12], and MuPlex [13], are neither multiplex programs nor are they free for all users. More

Table 1 Comparison of different primer design software programs

\begin{tabular}{|c|c|c|c|c|c|c|}
\hline & Ultiplex & PrimerPlex & MPD & Primer3 & Primer-BLAST & Oligo \\
\hline Multiplex PCR & Yes & Yes & Yes & No & No & No \\
\hline Input & BED & FASTA & BED & FASTA & FASTA & FASTA \\
\hline Web interface & Yes & No & No & Yes & Yes & No \\
\hline Free for academics & Yes & No & Yes & Yes & Yes & No \\
\hline Avoid SNPs in primers & Yes & Yes & Yes & - & No & No \\
\hline Avoid primers in repeat areas & Yes & No & Yes & No & No & No \\
\hline Single primer secondary structure check & Yes & Yes & No & Yes & Yes & Yes \\
\hline Specificity checking alignment algorithm in the whole genome & Yes & No & No & No & Yes & No \\
\hline Mutually compatible without secondary structure & Yes & Yes & No & - & - & - \\
\hline Mutual specificity checking alignment algorithm in the whole genome & Yes & No & No & - & - & - \\
\hline Self-defined species genome & Yes & No & No & - & Yes & - \\
\hline Next-generation sequence addition & Yes & Yes & Yes & - & - & - \\
\hline Graphic overview of primers found & Yes & - & No & No & Yes & Yes \\
\hline
\end{tabular}


importantly, multiplex PCR primer design software that can eliminate nonspecific amplification of the whole genome in multiplex PCR is rare and usually requires strenuous experimental validation.

Here, we developed the web-based multiplex PCR primer design software "Ultiplex". It was developed in the Python language with the Flask, Primer3 core and BLASTn+ command-line tools. Ultiplex provides the combined performance of Primer3 and BLASTn+ in a user-friendly interface on a high-performance computational platform. It not only designs primers but also evaluates the performance of different primer pairs in a single reaction and filters and clusters multiplex primers.

\section{Implementation}

\section{Ultiplex-core program}

The main primer design and calculation procedures are conducted with the Ultiplex-core Program at the service centre, which was programmed in the Python language with the Flask, Primer3 core and BLAST+ command-line tools. The Ultiplex-core is formed by four modules (Fig. 1): "InputF" for argument input, "Getprimers" for primer design and filtration, "Multiplex" for multiplex primer pair clustering, and "Report" for the graphic overview of primer design.

1) "InputF" arguments input module: This module accepts the parameters from the web interface and

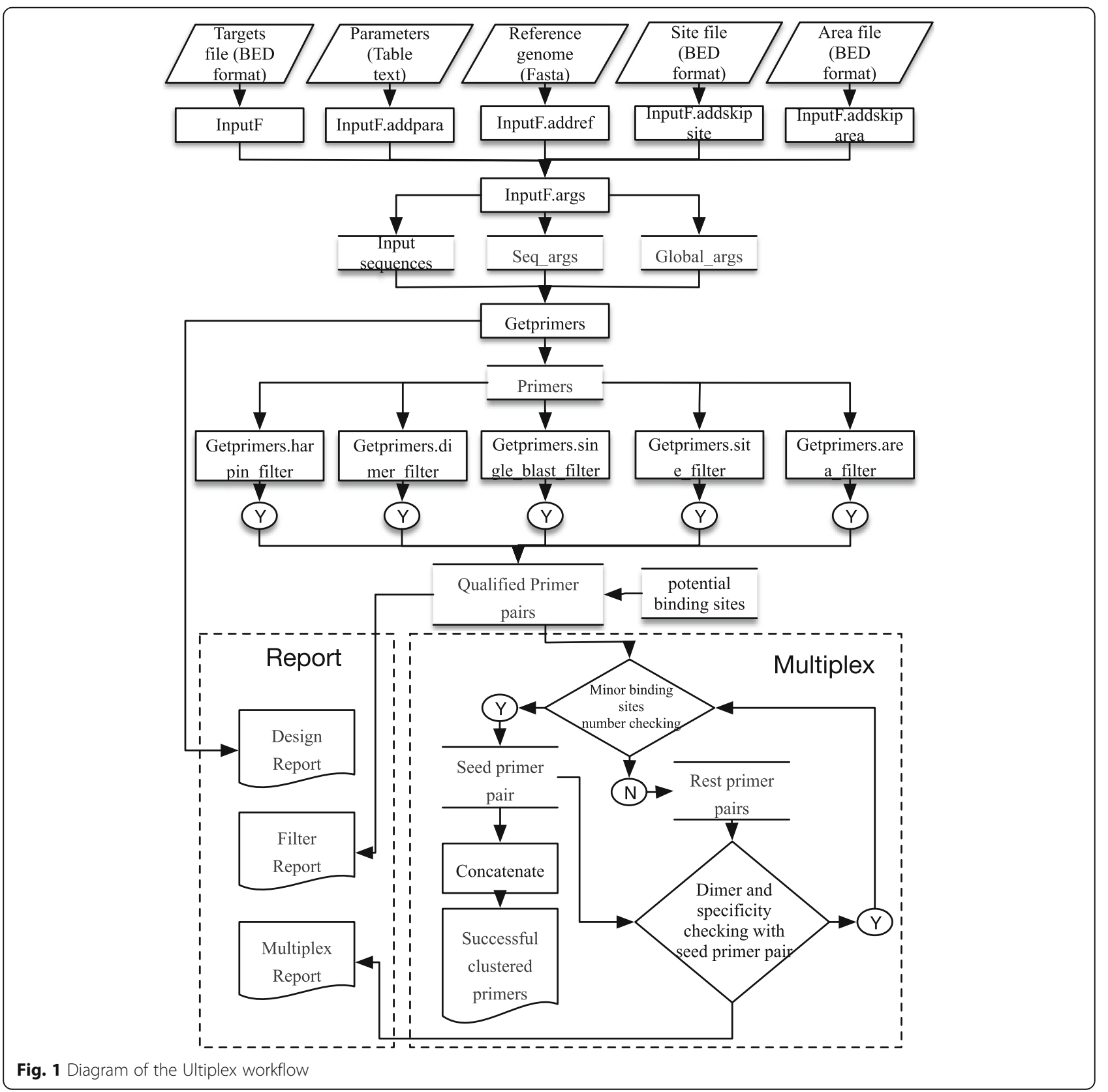


generates the arguments and environment for subsequent modules. The genome reference is indexed with BLASTn+ command-line tools [14], and BLASTn+ database files are generated. The "pybedtools" package [15] is used to generate target sequences from genome references in the range of [target start position - product max size +1 , target end position + product max size]. Seq_args and Global_args, needed by the Primer3 core, are also generated, including Tm values, product size, primer size, GC\% and primer numbers.

2) "Getprimers" primer design and filtration module: Primer pairs were designed with the primer-py package (https://github.com/libnano/primer3-py) and with target sequences and parameters from the previous module. The failed designed primers and reasons for failure were recorded. Secondary structures, such as hairpins and dimers, can affect primer amplification efficiency. Unlike other primer software, primers are individually checked for secondary structures. Under the "Getprimers.harpin_filter" function, primers and their $5^{\prime}$ tags are combined and tested with the "primer3.calcHairpin" function of primer-py, and primers showing hairpin secondary structures with $\mathrm{Tm}$ values over $45^{\circ} \mathrm{C}$ are eliminated (Fig. 2A). Under the

"Getprimers.dimer_filter" function, forward primers and reverse primers combined with their tags are compared to check dimer secondary structures with the "primer3.calcHeterodimer" function. Primers exhibiting dimer secondary structures whose Tm values are over $40^{\circ} \mathrm{C}$ will be eliminated (Fig. 2B). Under the "Getprimers.area_filter" and "Getprimers.site_filter" functions, if the final 7 bp sequence at the $3^{\prime}$ end of a primer is located in a skipped site (such as SNPs and other user-defined sites) or

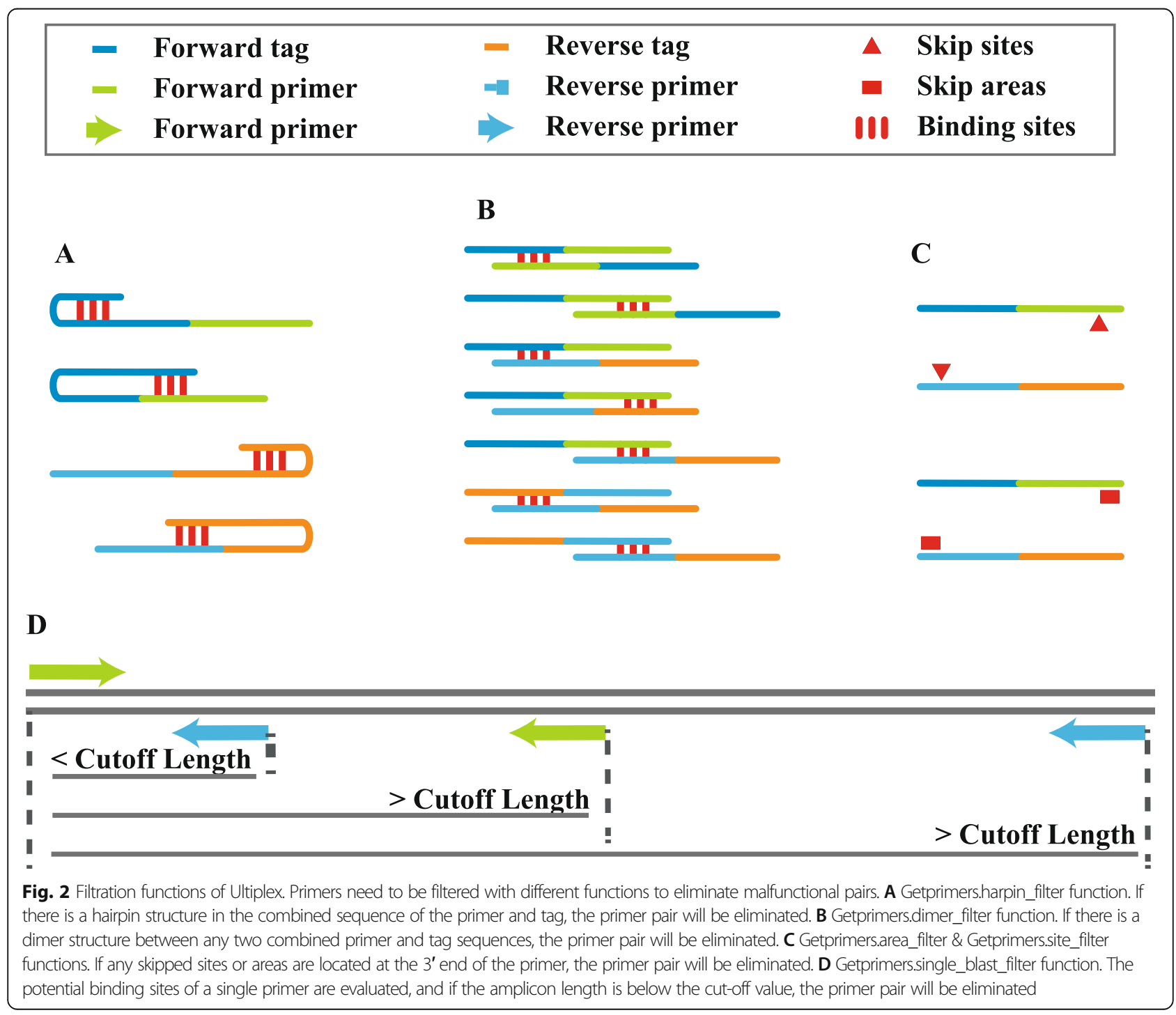


repeat area (such as repeats, tandem repeats, indels and other user-defined areas), it will be filtered out (Fig. 2C).

More importantly, the "Getprimers.single_blast_filter" function is to check primer pair specificity. The specificity of the primer pair (or unique amplification of the genome) is checked by aligning forward/reverse primers to the whole genome with BLASTn+ command-line tools and calculating the possible amplicons of primer pairs within the genome. A single genome sequence will be assumed to be the potential binding site of a single primer if the following conditions are met. 1) The aligned genome sequence is longer than $12 \mathrm{bp}$, and the BLASTn+ e-value is over 1000. 2) The delta G value between the primer and the aligned genome site is above the threshold. 3) The mismatch at the 3' end of each primer is smaller than $3 \mathrm{bp}$. 4) The mismatch number between the primer and aligned genome sites is smaller than $9 \mathrm{bp}$. If the distance between the potential binding site of the forward-reverse primer, forward-forward primer or reverse-reverse primer for one primer pair is below the threshold, the paired alignments will be assumed to be possible amplicons of the primers (Fig. 2D). When these possible amplicons are located outside of our target area, they will be assumed to be false-positive amplicons, and the related primer pairs will be filtered out. Throughout the process, the primer3-py package is used for delta $\mathrm{G}$ calculation.

3) "Multiplex" for multiplex primer pair clustering: With the "Multiplex" function, the unity and incompatibility between different pairs are tested for each pair, and compatible pairs are clustered. Unity refers to product length and Tm unity. The difference in the length of the two primer pairs should be less than $150 \mathrm{bp}$, and the difference in Tm between the two primer pairs should be less than $5^{\circ} \mathrm{C}$. Incompatibility refers to dimers and nonspecific alignments generated between different pairs. The checks for dimers and nonspecific alignment are described above. The only difference is that these tests are conducted between different pairs. As the relationships between pairs are deduced, the list of maxim unifiable and compatible primers is generated.

4) "Report" graphic overview of primer design: The primer design, filtering and clustering results can be graphically illustrated by using "Report" functions, as can the failed targets and reasons for failure. The graphs are generated with the Python package pyecharts (https://github.com/pyecharts/pyecharts) and are illustrated for the user in the detailed project information interface (Fig. 3B).
Tested variant list and primer design

We chose 250 hotspot mutations associated with HNPC $\mathrm{C}$ and hepatocellular carcinoma and 45 chemotherapy cytotoxicity-related variants (Table S1) as design targets to test Ultiplex. Primers (Table S2) were designed with Ultiplex following the provided guide.

\section{Cell collection and DNA extraction}

HCT-15 and HaCaT cells were obtained from the Fifth Affiliated Hospital of Southern Medical University. The cells were centrifuged at $3000 \mathrm{~g} / \mathrm{min}$. DNA extraction was conducted with a Qiagen mini kit (Germany).

\section{Primer amplification and library construction}

DNA was fragmented into pieces smaller than $500 \mathrm{bp}$ with a Bioruptor ${ }^{\odot}$ Pico instrument (Diagenode SA). The first round of PCR amplification was conducted with $10 \mu \mathrm{L}$ of master mix (Vazyme, Nanjing, China), $1 \mu \mathrm{L}$ of fragmented DNA $(50 \mathrm{ng} / \mu \mathrm{l}), 1 \mu \mathrm{L}$ of multiplex primer mix (the final concentration of each primer pair was 0.5 $\mathrm{nM})$ and $8 \mu \mathrm{L}$ of distilled deionized water (ddH2O). The first round of PCR amplification started at $94{ }^{\circ} \mathrm{C}$ for 5 min, followed by 15 cycles of $94{ }^{\circ} \mathrm{C}$ for $5 \mathrm{~s}, 60^{\circ} \mathrm{C}$ for $30 \mathrm{~s}$, $72{ }^{\circ} \mathrm{C}$ for $20 \mathrm{~s}$, and then a final step at $72^{\circ} \mathrm{C}$ for $5 \mathrm{~min}$. The products were purified with AMPure XP beads (Agencourt, MA, USA). An $8 \mu \mathrm{L}$ aliquot of the purified products was amplified with $10 \mu \mathrm{L}$ of master mix (Vazyme, Nanjing, China), $2 \mu \mathrm{L}$ of modified i5 and i7 primer mix (the final concentration of each primer was $10 \mathrm{nM}$ ) (Table S3). The thermal cycling program started at $94{ }^{\circ} \mathrm{C}$ for $30 \mathrm{~s}$, followed by 12 cycles of $94{ }^{\circ} \mathrm{C}$ for $5 \mathrm{~s}$, $60{ }^{\circ} \mathrm{C}$ for $30 \mathrm{~s}$, and $72{ }^{\circ} \mathrm{C}$ for $20 \mathrm{~s}$. The pooled products were purified with AMPure XP beads (Agencourt, MA, USA) and qualified on an Agilent 2100 Bioanalyzer (Agilent Technologies, Santa Clara, CA, USA) and a Qubit 3.0 Fluorometer (ThermoFisher Scientific, Waltham, MA, USA).

\section{Sequencing and analysis}

The libraries were sequenced with a NovaSeq 6000 System (Illumina). The sequencing depth for each target was over 1000x. After sequencing was complete, raw data were assigned to each sample ID based on the barcode of the i5 and i7 primers. After low-quality read clearance by using fastp [16], clean reads were mapped to the reference sequence by using Bowtie 2 [17] with the parameter "--very-sensitive". Only paired-end mapping reads were retained for SNP calling. SNP calling was conducted with SAMtools [18] and BCFtools [19].

\section{Results and discussion}

Web-based interface

Ultiplex was developed as an online multiplex primer design tool with a user-friendly web-based interface 


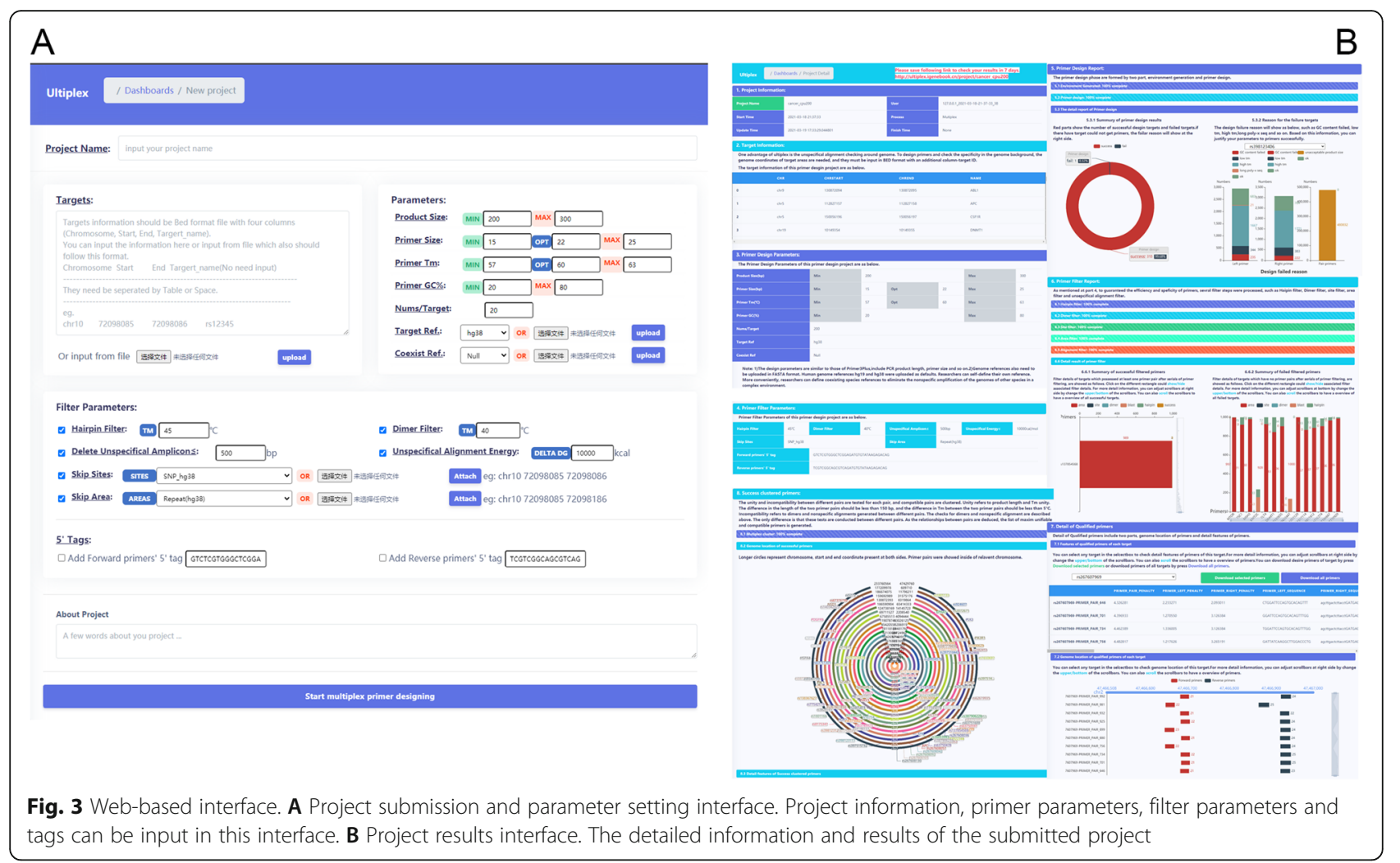

programmed with Flask, SQLite and Python software and other packages. There is no need for users to possess programming skills or construct a design environment to use Ultiplex, meaning that users (especially researchers) can easily design primer panels. The design parameters are similar to those of Primer3Plus [20]. There are differences in terms of the target input, reference file upload, skipped sites and areas defined and primer $5^{\prime}$ tags defined (Fig. 3A).

1) To design primers and check the specificity in the genome background, the genome coordinates of target areas are needed, and they must be input in BED format with an additional column-target ID. 2) Genome references also need to be uploaded in FASTA format. Human genome references hg19 and hg38 were uploaded as defaults. Researchers can self-define their own reference. More conveniently, researchers can define coexisting species references to eliminate the nonspecific amplification of the genomes of other species in a complex environment. 3) Researchers can define sites and areas that need to be skipped. There are two main categories of sequences that need to be masked: areas (repeats/tandem repeats/indels) and sites (SNPs and other small variances). PCR performance will be affected if primers are located in these areas. When primers are located in repeat areas, nonspecific amplification may occur. When the 3' end of the primers is located in areas with a small amount of variance, PCR amplification of the variable sequences may fail. Additionally, users may need to exclude some areas due to their own needs. 4) For further analysis and sequencing of the product, sequencing primers need to be added to the $5^{\prime}$ ends of the library, and researchers can choose different strategies, such as "adapter ligation" [21] or "two-step PCR" [22]. If researchers choose to use the "two-step PCR" strategy, $5^{\prime}$ tags need to be added at the $5^{\prime}$ end of the primers, and we provide one pair of tags for the Illumina sequencing platform as the default: GTCTCGTGGGCTCGGA GATGTGTATAAGAGACAG and TCGTCGGCAGCG TCAGATGTGTATAAGAGACAG. Researchers can define tags according to their own needs. Once parameters are submitted, a project is created and processed at our service centre. Progress will be updated as shown in Fig. 3B. With a simple click, detailed project information will be provided indicating the detailed design progress and completed design results.

\section{Comparison to other primer design tools}

For primer design functions, Ultiplex offers several advanced functions that are not available in other software tools. Table 1 provides a brief summary of these functions, many of which are important for multiplex primer design. For example, Ultiplex is the only tool that offers the ability to examine the mutual compatibility of 
clustered multiplex primers in the whole genome. It is difficult to avoid nonspecific amplification when we simply mix the specified single primer pairs together because there may be false amplification between different pairs. The probability of nonspecific amplification will increase as PCR multiplicity increases. To avoid nonspecific amplification, Ultiplex can check the specificity between different primer pairs in the whole genome with BLASTn+ command-line tools and can check for secondary structures between different primer pairs. The compatible primer pairs will be clustered together, and primers for other targets will be redesigned or allocated into other clusters based on the user settings. Furthermore, users can define their own genome references and areas that they need to skip. This option is useful for microbe and pathogen researchers because their targets exist in complicated environments, including host and other microbe genomes. With Ultiplex, false amplification in other species can easily be avoided. Additionally, detailed design features, reasons for failure and cluster information are illustrated with graphic reports in Ultiplex.

For the principles behind primer design tools, BLASTn+ command-line tools and primer3 were reported to be used separately or combined for primer design, or even multiplex primer design in several studies. BatchPrimer3 was developed with Primer3 core and BLASTn+ for microsatellite (simple sequence repeat-SSR) and single nucleotide polymorphism marker primers [23]. However, BatchPrimer3 is mainly based on an allele-specific PCR strategy and cannot be used for multiplex primers. ThermoAlign is a tiled amplicon (long range sequence) resequencing primer design tool used in Primer3 core and BLASTn+, but it cannot be used for special sites or areas [24]. Yaheng Wang developed a multiplex PCR primer design system for targeted sequencing, which is similar to combining Ultiplex with Primer3-py and BLASTn+ [25]. However, this multiplex PCR primer design system lacks the ability to examine the mutual compatibility of clustered multiplex primers within the whole genome and is not available to all users.

\section{Thresholds of nonspecific alignments and secondary structures}

Hairpins, dimers and nonspecific alignments are three important parameters in the analysis of the specificity of single primer pairs and mutual primer compatibility. Tm and delta $G$ are the main parameters used to determine whether harmful hairpins, dimers and nonspecific alignments may occur. We employed the nearest neighbour thermodynamic parameters of primer3 to calculate Tm and delta G. The default formulations of $\mathrm{NaCl}, \mathrm{Mg} 2+, \mathrm{DMSO}$, and dNTPs were described previously [26]. The Tm and delta G thresholds of hairpins, dimers and nonspecific alignments were set at $45^{\circ} \mathrm{C}, 40^{\circ} \mathrm{C}$ and $-10 \mathrm{kcal} / \mathrm{mol}$, respectively, following a previous study [27]. Users are free to define thresholds for hairpins, dimers and nonspecific alignments before the design procedure. Five sets of single primers with different delta $G$ cutoff values were tested. Each set of primers (Table 2) contained 10 single primers that represented the possible false amplicon at each delta $G$ threshold (Fig. 4A). The accurate amplification rate (Fig. 4F and G) of primers with delta $G$ values above $-10 \mathrm{kcal} / \mathrm{mol}$ was $100 \%$. For primers with delta $G$ values of -10 $-11,-11 \sim-12,-12 \sim-13$ and under $-13 \mathrm{kcal} / \mathrm{mol}$ (Fig. 4B-F), the accurate amplification rates were 90, 70,70 , and $70 \%$, respectively. This result shows that the default delta $G$ threshold is sufficient to distinguish nonspecific alignments under our recommended PCR conditions.

\section{Performance and results of primer design and single- target filtering}

A total of 295 targets were designed with Ultiplex, among which $99.7 \%$ of sites were designed successfully, and 1 target (rs398123406) showed design failure (Fig. 5A). Product size is the main reason for failure. As shown in Fig. 5B, a total of 315 left primers and 336 right primers were designed successfully, but the left primers and right primers were not matched with each other according to the appropriate length. After checking the detailed input information, we found that the rs398123406 target was 450 bp in length (Table S1). An overly broad target range results in fewer choices for primer design. A narrower target range will correct the design result.

Nineteen of 294 successfully designed targets failed according to primer filtration (Fig. 5C). A location in a skipped area was the main cause of filtration, and 17 target primers located in such areas had to be skipped (these areas are actually repeat-sequence areas in the human genome according to our default setting). The other reasons for filtration were the identification of dimers or skipped sites and specificity in the genome. A total of $5.83 \%$ of the primer pairs were filtered out because of the specificity checks. The qualified primer pairs were generated and could be saved to local files with unique alignments (Fig. 5D, Table S5). Design and filtering information can be generated with the "Report" functions of Ultiplex. The resultant graphics have partial interaction functions and can provide to users to help them adjust their target areas and parameters during redesign based on information such as the identification of secondary structures, repeat areas, false alignments and so on. 
Table 2 Delta G cut-off values for primer sets

\begin{tabular}{|c|c|c|c|c|c|c|}
\hline deltaG cut-off & Primer names & Primer_sequences & $\mathrm{Chr}^{*}$ & Length & $\begin{array}{l}\text { Left_site } \\
\text { deltaG }^{\Delta}\end{array}$ & $\begin{array}{l}\text { Right_site } \\
\text { deltaG" }\end{array}$ \\
\hline \multirow{10}{*}{$\begin{array}{l}<-13 \mathrm{k} \\
\mathrm{cal} / \mathrm{mol}\end{array}$} & T13k_1 & GGCCTGGCTCTCTATACCCC & 6 & 325 & $-13,223$ & $-15,028$ \\
\hline & T13k_2 & ACGTTGCTCCCGCTCACC & 16 & 945 & $-14,693$ & $-14,693$ \\
\hline & T13k_3 & AAGACCACGAGGCACAGC & 20 & 1778 & $-13,580$ & $-13,372$ \\
\hline & T13k_4 & CCACAGCATCGTGACCCTG & 2 & 1453 & $-15,972$ & $-15,972$ \\
\hline & T13k_5 & AGCAAGGAAGTGGACAGGTC & 8 & 572 & $-13,149$ & $-13,149$ \\
\hline & T13k_6 & GTGTAGGAGCTGCGCCTC & 5 & 503 & $-13,483$ & $-13,420$ \\
\hline & T13k_7 & ATCTGCAGCAGTTTGGAGCC & 6 & 742 & $-13,454$ & $-13,454$ \\
\hline & T13k_8 & TCTCCACGATGCCGGCTG & 9 & 1402 & $-13,003$ & $-14,414$ \\
\hline & T13k_9 & GTTTCTTCTCCATCGCGGGG & 17 & 1130 & $-13,847$ & $-13,573$ \\
\hline & T13k_10 & CTCCATGGCTGATCTCCCCT & 16 & 1736 & $-13,195$ & $-13,195$ \\
\hline \multirow{10}{*}{$\begin{array}{l}-12 \sim-13 \mathrm{k} \\
\mathrm{cal} / \mathrm{mol}\end{array}$} & T12k_1 & CAGATCACCTACAGCGCCA & 11 & 1635 & $-12,176$ & $-12,176$ \\
\hline & T12k_2 & TAACCCCCATAGCCCTCAAC & 1 & 1645 & $-12,460$ & $-12,621$ \\
\hline & T12k_3 & CATATGGCCGTGTCTGGGG & 2 & 239 & $-12,572$ & $-12,021$ \\
\hline & T12k_4 & CCAGCCCAGCATTCTCCAG & 19 & 1864 & $-14,841$ & $-12,519$ \\
\hline & T12k_5 & GCAGCAGGGATTGGATGGC & 15 & 1776 & $-12,218$ & $-12,218$ \\
\hline & T12k_6 & GCAGGGATTGGATGGCCC & 11 & 855 & $-12,436$ & $-12,115$ \\
\hline & T12k_7 & CATCTTCGATTTGAGTGCCCC & 12 & 1868 & $-12,150$ & $-12,150$ \\
\hline & T12k_8 & TTCTTGAAGAGGACGGTGCC & 2 & 1298 & $-12,580$ & $-12,380$ \\
\hline & T12k_9 & TCTGAGCATTTGGGCTTGC & 3 & 984 & $-12,562$ & $-12,671$ \\
\hline & T12k_10 & CAAGATGTCGGGGAGTGGCC & 17 & 1502 & $-12,976$ & $-12,900$ \\
\hline \multirow{10}{*}{$\begin{array}{l}-11 \sim-12 \mathrm{k} \\
\mathrm{cal} / \mathrm{mol}\end{array}$} & T11k_1 & GGCTAAGGGGGTACACTTCA & $x$ & 657 & $-11,112$ & $-11,112$ \\
\hline & T11k_2 & CTTGGAAGTGGACGTAGGTGT & $x$ & 802 & $-11,333$ & $-11,333$ \\
\hline & T11k_3 & GGTGTCTGTCCCGGCCAG & 4 & 529 & $-13,905$ & $-11,348$ \\
\hline & T11k_4 & CATTTACAATCCAGCCAAATCACT & 7 & 1475 & $-11,879$ & $-11,879$ \\
\hline & T11k_5 & GCTCCTGGGGCATAGGGATG & 3 & 335 & $-11,911$ & $-11,911$ \\
\hline & T11k_6 & CAGGGATTGGATGGCCCC & 11 & 516 & $-11,193$ & $-11,193$ \\
\hline & T11k_7 & CTGGCTTGGCACTGGTCT & 15 & 916 & $-11,926$ & $-11,322$ \\
\hline & T11k_8 & AGCCCTGTGTGGTTCCAAG & 6 & 916 & $-11,194$ & $-11,175$ \\
\hline & T11k_9 & CTCACTCCATCCTGGACGT & 2 & 452 & $-11,110$ & $-11,110$ \\
\hline & T11k_10 & GCTCACTCCATCCTGGACGT & 2 & 452 & $-11,868$ & $-11,868$ \\
\hline \multirow{10}{*}{$\begin{array}{l}-10 \sim-11 \mathrm{k} \\
\mathrm{cal} / \mathrm{mol}\end{array}$} & T10k_1 & СCTCCTTGTGCCCTAGAGTT & 14 & 185 & $-10,271$ & $-10,271$ \\
\hline & T10k_2 & cctttctgttgcctGTGGTAAG & 5 & 161 & $-10,543$ & $-10,224$ \\
\hline & T10k_3 & GCTTITCCTCATGGGGCAAA & 4 & 447 & $-10,575$ & $-10,809$ \\
\hline & T10k_4 & CCAGGACAAGGCCTCATCC & 4 & 325 & $-10,242$ & $-10,853$ \\
\hline & T10k_5 & TTCCATGGCAGACTTGTAAGGA & 14 & 401 & $-10,934$ & $-10,934$ \\
\hline & T10k_6 & CAGTGTGGTAAAAACGTCAGGATG & 2 & 464 & $-10,167$ & $-10,167$ \\
\hline & T10k_7 & AGTTGCAGAACCTTGCCCT & 14 & 375 & $-10,812$ & $-10,350$ \\
\hline & T10k_8 & TCGAGACGGGGGTAAAGGAG & $x$ & 147 & $-10,799$ & $-10,073$ \\
\hline & T10k_9 & AGGAATTCATGCCTITGGGACA & 6 & 191 & $-10,203$ & $-10,678$ \\
\hline & T10k_10 & CTGCTCCATGGCTGATCTCC & 4 & 316 & $-10,235$ & $-10,809$ \\
\hline \multirow{3}{*}{$\begin{array}{l}-9 \sim-10 \mathrm{k} \\
\mathrm{cal} / \mathrm{mol}\end{array}$} & T9k_1 & ATGGAGGCTGGATAGGAGGT & 1 & 162 & -9516 & -9084 \\
\hline & T9k_2 & GCCTCCTTGTGCCCTAGAGT & 14 & 187 & -9598 & -9598 \\
\hline & T9k_3 & GGGTCTTGGCAAGTGGTACTG & 6 & 394 & -9068 & -9307 \\
\hline
\end{tabular}


Table 2 Delta G cut-off values for primer sets (Continued)

\begin{tabular}{lllllll}
\hline deltaG cut-off & Primer names & Primer_sequences & Chr $^{*}$ & Length & $\begin{array}{l}\text { Left_site } \\
\text { deltaG }^{{ }^{*}}\end{array}$ & $\begin{array}{l}\text { Right_site } \\
\text { deltaG }^{\mathbf{*}}\end{array}$ \\
\hline & T9k_4 & GCTCCTGGGGCATAGGGAT & 3 & 337 & -9737 & -9737 \\
& T9k_5 & GATTCAGGGTGGTCACAGT & 2 & 419 & -9299 & -9050 \\
& T9k_6 & ACGCTGCTATGGTTCTACAG & 17 & 276 & -9320 & -9506 \\
& T9k_7 & GCAGCAGGGACTCACCATTG & 2 & 409 & -9396 & -9861 \\
& T9k_8 & ATTCAGCGTGATGTGTAATGAGG & 9 & 428 & -9135 & -9334 \\
& T9k_9 & AGAGGCTCCTGCAATTGACAG & 9 & 302 & -9394 & -9330 \\
& T9k_10 & TCAGACCAGTGCTACCTGAGAG & 9 & 202 & -9438 & -9577 \\
\hline
\end{tabular}

*: Chromosome number, $\Delta$ : Delta G value (cal/mol) of Left aligned site of the primer on the possible false amplicon, $\mathbf{m}$ : Delta $\mathrm{G}$ value (cal/mol) of right aligned site of the primer on the possible false amplicon

\section{Multiplex primer clustering results}

Among 275 targets, 261 were successfully clustered into one compatible PCR group. These 261 targets could be covered with 98 primer pairs due to the overlap between targets and primers (Fig. 6). Information on this overlap was also generated (Fig. 7). For example, rs267607850460 overlapped with 4 targets (rs267607853, rs63751657 and 2 other targets), which means that these 4 targets were located in the primer pair rs267607850-460 amplicon. With the advantage of the single-nucleotide

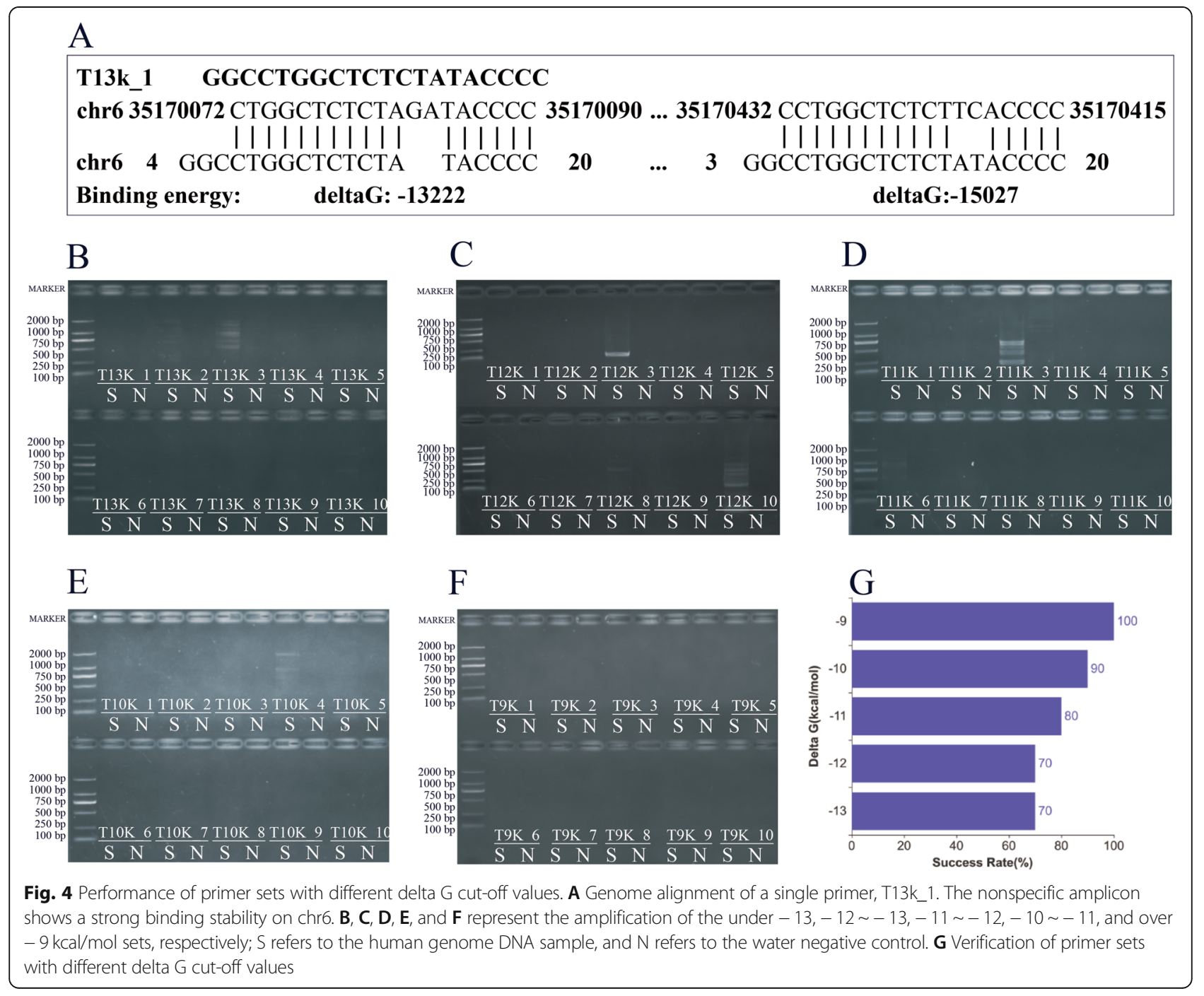




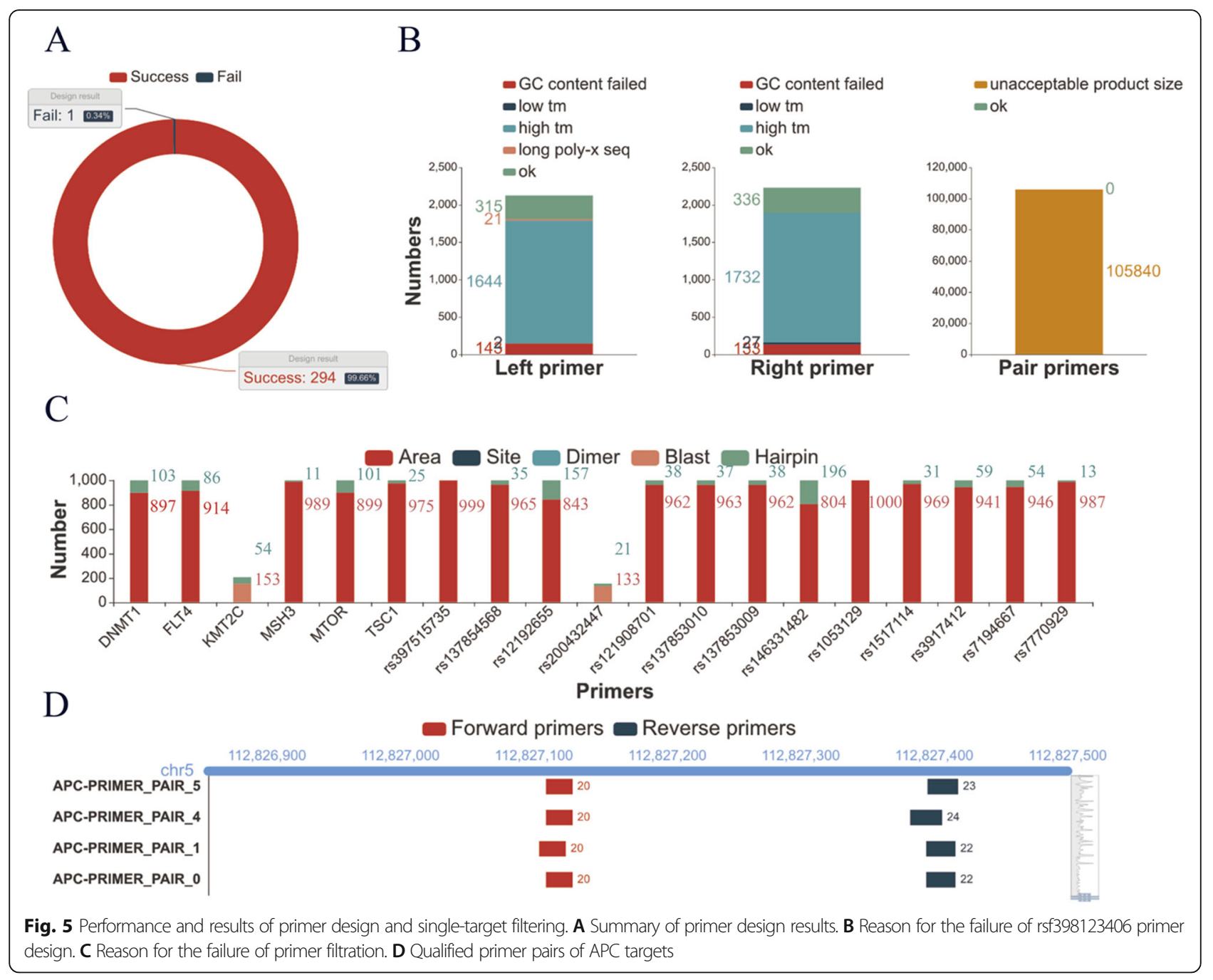

resolution of next generation sequencing (NGS), we could obtain polymorphism information not only for rs267607850 but also for the 4 other targeted polymorphisms. There was no need to design primers for these overlapping targets. Fourteen of 275 targets could not be integrated into this group because of secondary structures and false alignments, for which false alignment was the main reason.

As shown in Fig. 8A, 99.6\% of the primer pairs of targets that failed to be integrated into the compatible group showed false alignment with primer pairs in this compatible PCR group (Fig. 8B). Six of 14 targets possessed at least one primer pair that showed false alignment with only one primer pair in the compatible $\mathrm{PCR}$ group (Fig. 8B/C). The total target number of the compatible group increased as we lowered the threshold for the number of false alignments between primer pairs in this group (Fig. 8D). When we allowed there to be 1 false alignment between 14 incompatible target primers and the $98 \mathrm{com}$ patible primer pairs, 5 pre-incompatible targets could be clustered in the compatible PCR group. When we allowed there to be 4 false alignments between those two groups, 10 pre-incompatible targets could be clustered in the compatible PCR group. As a result, 271 targets were included in a single primer cluster covered by 108 primer pairs (Fig. 6), and 4 targets (rs267607950, rs267607953, rs16857540, and rs716274) were incompatible with this cluster. Limited nonspecific amplification events are acceptable in next-generation sequencing PCRs, particularly for projects with a sufficient budget, because these amplicons can be distinguished clearly with next-generation sequencing and bioinformatic pipelines.

\section{Increased multiplex clustering speed and time and storage consumption}

Although multiplex cluster simulation could simplify tedious experimental library generation work and reduce the time requirement by months, the calculation process may be longer because of computer storage, CPU and argument settings. The multiplex clustering calculation 


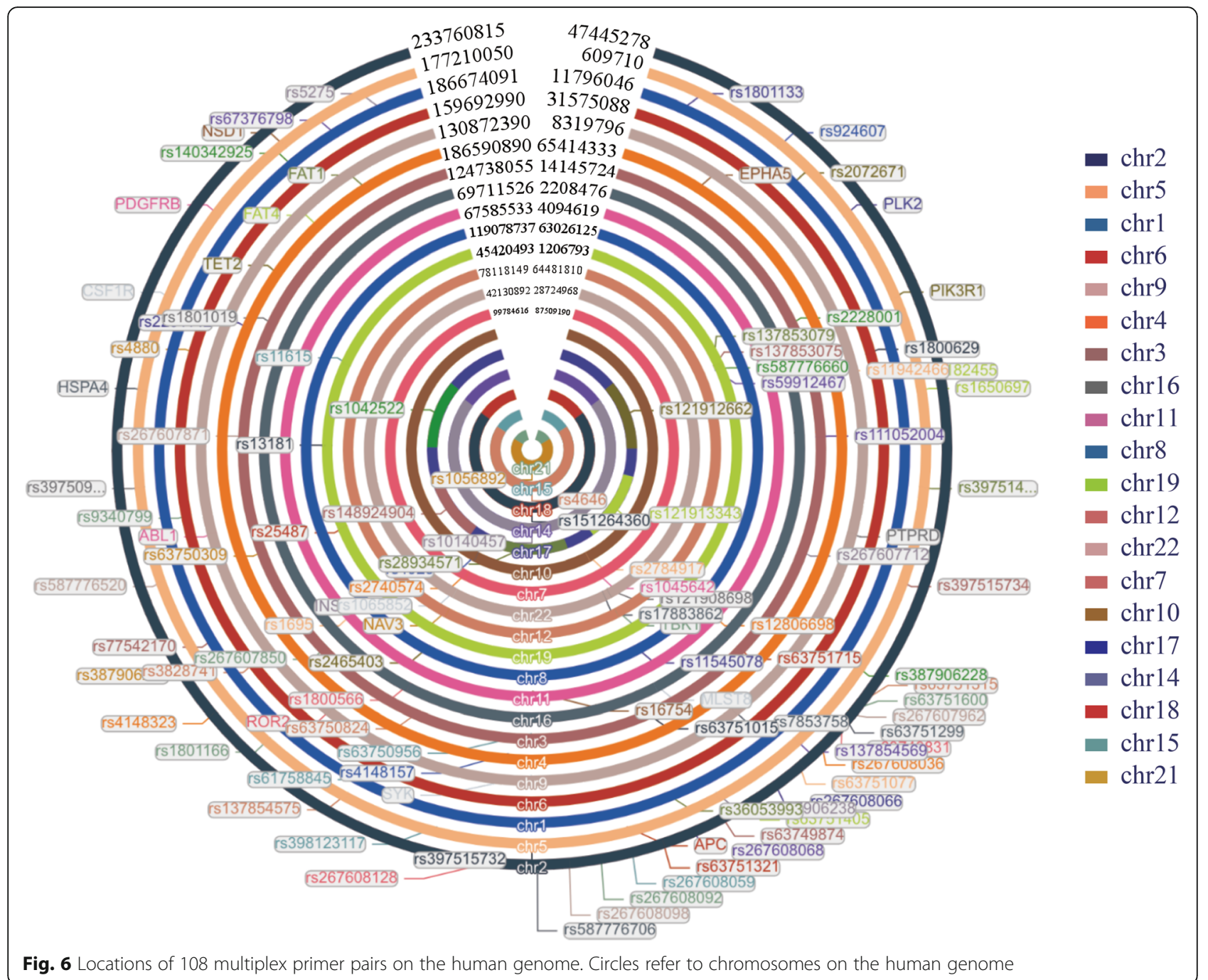

step is the most memory-intensive and time-consuming calculation step in multiplex primer design, especially when the number of primers for each target is larger. When we needed to obtain a compatible relationship between $\mathrm{m}$ target primer pairs, we assumed that each target had $\mathrm{n}$ primer pairs and that both primers in one primer pair had $k$ nonspecific sites on average; the multiplex cluster calculation was as follows: $\complement_{m}^{2} \times 4$ $\times C_{n \times k}^{1} \times C_{n \times k}^{1}$ (Fig. 9A). To eliminate complexity and speed up the process, we assumed that one primer pair (seed primer pair), which possessed minimal nonspecific binding sites, showed the highest possibility of appearing in our ideal mutually compatible cluster. In each turn, one seed primer pair was selected from all primer pairs of all targets based on the number of nonspecific binding sites. The primer pairs for the remaining targets that were incompatible with this seed primer pair were deleted. The next seed primer pair was selected from the remaining primer pairs. After several iterations, when no primer pairs remained, the pool of all seed primer pairs was our ideal compatible multiplex primer cluster. Thus, the calculation procedure becomes much simpler (Fig. 9B).

After increasing the speed of the procedure, it was tested for time and computer storage consumption with different tasks. As shown in Table 3, when the initial primer number per target was two, Ultiplex required $1.3 \mathrm{~h}$ to design primers for 295 targets. When the initial primer number per target was increased to 100 , the time required was $14.7 \mathrm{~h}$. The complexity of the calculation process increases as the initial primer pair number (n) for one target or the target number $(\mathrm{m})$ increases.

\section{Multiplex PCR process and sequencing results}

We modified the multiplex experimental strategy of Genotyping-in-Thousands by sequencing (GT-seq) with a previous template fragmentation step (Fig. 10A) to increase multiplicity. When we fragmented the DNA 


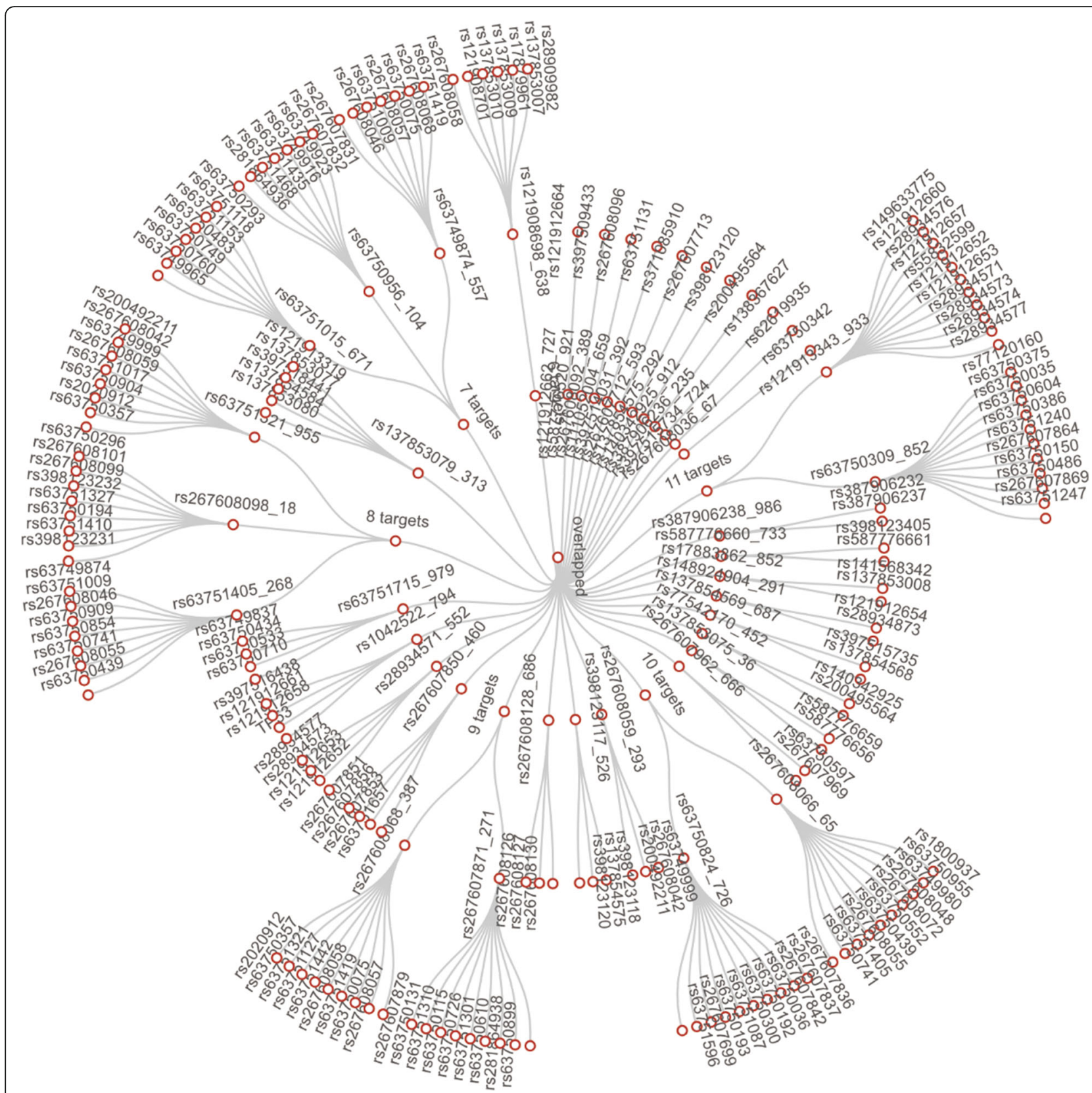

Fig. 7 Summary of the overlap between successfully clustered sites

template to a size smaller than $500 \mathrm{bp}$, the multiplicity was increased from 68-plex to 98-plex. The improvement was caused by the eradication of nonspecific amplicons over $500 \mathrm{bp}$ between multiple primer pairs. Rather than template fragmentation, altering the PCR extension time is the common strategy for controlling amplicon length and nonspecific amplicon length. For example, a 30-s PCR extension step with regular DNA Taq polymerase is predicted to amplify a $500 \mathrm{bp}$ product and to reduce the generation of nonspecific amplicons longer than $2000 \mathrm{bp}$. However, due to the uncertainty of polymerase efficiency, this strategy cannot eradicate nonspecific amplicon production. With prior template fragmentation and our pairwise process for checking possible primer-genome amplicons smaller than $500 \mathrm{bp}$, 108-plex multiplex reactions can now be performed with high specificity.

A single primer pair showed a $93 \%$ amplification success rate (Fig. S1), and the multiplex PCR products were within the expected range (Fig. 10B). A total of 106 primer pairs could produce clear products, and 2 primer pairs produced faint minor products when used 


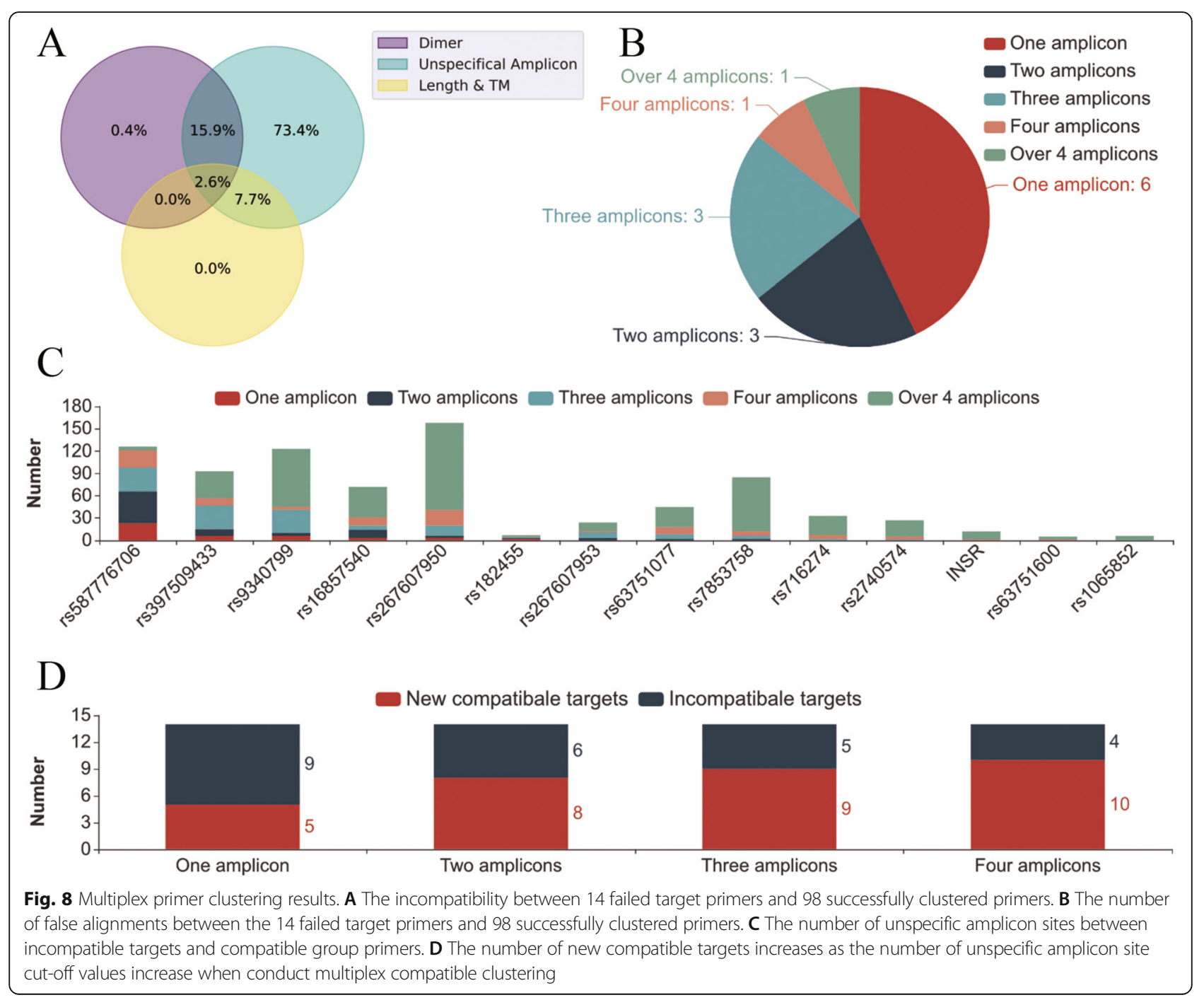

separately for the amplification of human DNA. A total of 108 primer pairs showed $100 \%$ specificity. These primers were specific to the human genome, as they generated unique products from human DNA. They also exhibited no secondary structures, such as dimers, as no products were generated from water. When the 108 primer pairs were pooled and amplified with a dedicated experimental workflow, the products were within our expected 200-500 bp range (Fig. 10B).

Two hundred megabase pairs of sequencing data per sample was generated with the 108 primer pairs. The average coverage of the 108 primer pairs was $5000 \mathrm{X}$, and the alignment rate was $99.21 \%$. Nonspecific amplicons were rarely generated, even though 10 nonspecific primer pairs were included in the reaction. Only 7 targets showed below 30X coverage (Fig. 10D), and as we doubled the concentration of the primers, the read coverage increased rapidly (Fig. 10C, Table S4). The performance of those low-efficiency primers in the multiplex PCR primer group could be enhanced by increasing the primer concentration.

\section{Sensitivity of variant detection}

DNA from HCT-15 and HaCaT cell lines was analysed with the Ultiplex associated experimental and bioinformatic workflow. Information for $245 \mathrm{SNPs}$ /variants was generated. In these $245 \mathrm{SNPs} /$ variants, Rs28934573 of the TP53 gene is significantly different between HCT-15 and $\mathrm{HaCaT}$ cell lines. This variant was tested to determine the detection limit and sensitivity of this system with a series of samples with different HCT-15 and HaCaT DNA ratios. The results show that multiplex PCR does not affect the sensitivity of single target detection. For rs28934573(C > T) (Table 4), this multiplex system stably detected mutation rates as low as $0.25 \%$. These rates were $0.25,0.29$ and $0.19 \%$ for the simulated sample with a $0.25 \%$ theoretical value. The coefficient of variation was $20.68 \%$. These results show that variant 


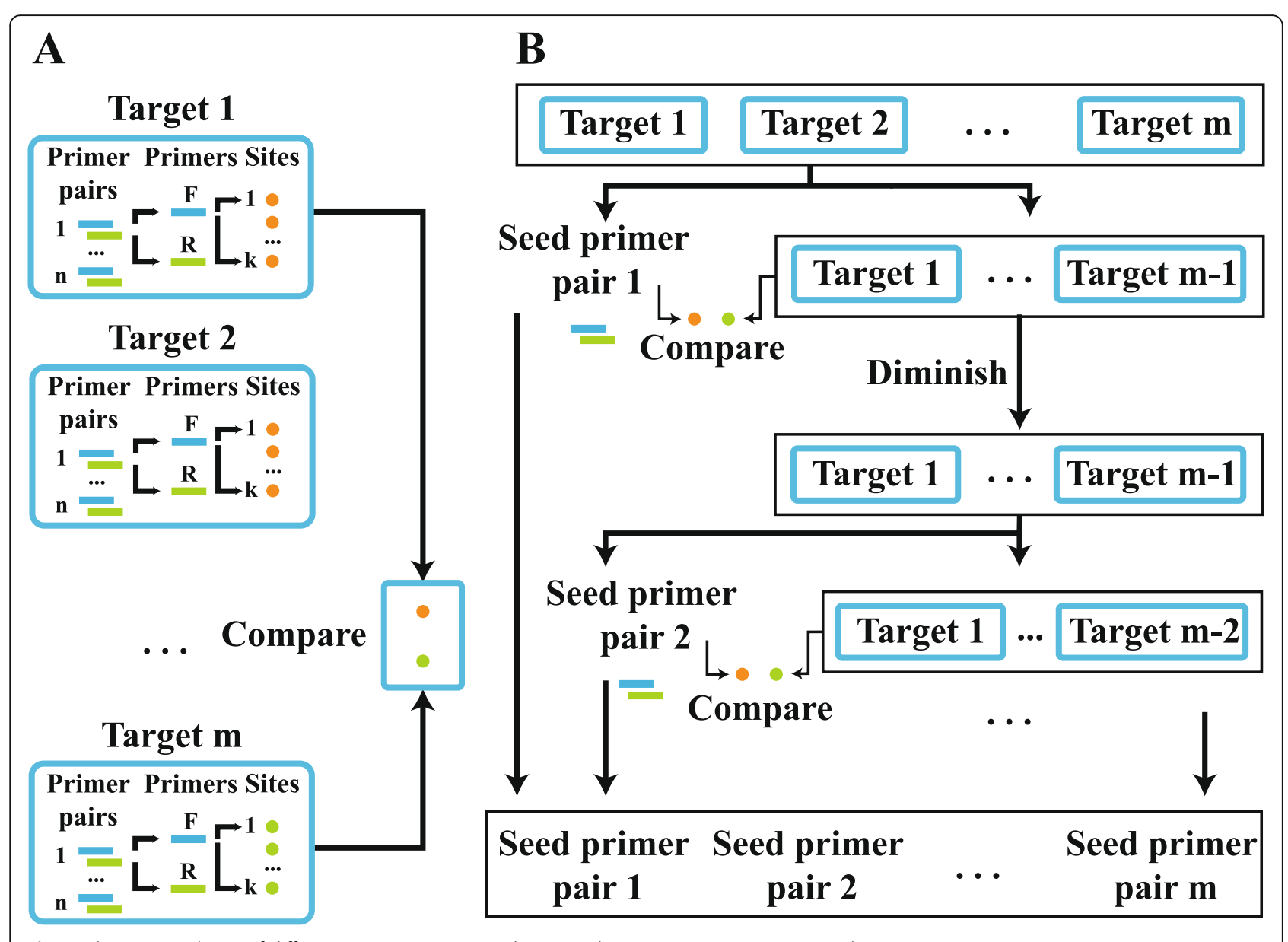

Fig. 9 Cluster complexity of different strategies. A Normal contrast between every primer. B Seed primer pair strategy

detection with the 'Ultiplex' multiplex primer group and associated experimental workflow is sensitive, stable and reproducible.

\section{Conclusions}

Ultiplex is a web-based multiplex PCR primer tool. With the associated experimental workflow, Ultiplex can be used to detect any genomic area with next generation sequencing for a species with a clear reference genome, including genotyping, disease associated variation detection, and species identification. Ultiplex has several functions, including batch design and compatibility checking for the exclusion of mutual secondary structures and mutual false alignments across the whole genome. It offers flexible arguments for users to define their own references, primer Tm values, product length, plex number and tag oligos. The input can be any BED file from any species reference. It can avoid repeat regions and variance sites at the end of primers to achieve precise amplification as well as those areas that users want to exclude. Finally, based on graphic primer design reports, users can not only design and generate highly specific multiplex PCR primers with Ultiplex but can also

Table 3 Consumption of time and storage of 295 target primers

\begin{tabular}{llllll}
\hline & $\begin{array}{l}\text { 2 primer pairs/ } \\
\text { target }\end{array}$ & $\begin{array}{l}\text { 20 primer pairs/ } \\
\text { target }\end{array}$ & $\begin{array}{l}\mathbf{5 0} \text { primer pairs/ } \\
\text { target }\end{array}$ & $\begin{array}{l}\text { 100 primer pairs/ } \\
\text { target }\end{array}$ & $\begin{array}{l}\text { 200 primer pairs/ } \\
\text { target }\end{array}$ \\
\hline Time & $1.3 \mathrm{~h}$ & $4 \mathrm{~h}$ & $8 \mathrm{~h}$ & $14.7 \mathrm{~h}$ & $18 \mathrm{~h}$ \\
Memory & $65 \mathrm{G}$ & $65 \mathrm{G}$ & $65 \mathrm{G}$ & $65 \mathrm{G}$ & $65 \mathrm{G}$ \\
CPU core & 32 & 32 & 32 & 32 & 32 \\
$\begin{array}{l}\text { Multiplicity of } \\
\text { Cluster }\end{array}$ & 56 & 85 & 97 & 98 & 103 \\
\hline
\end{tabular}




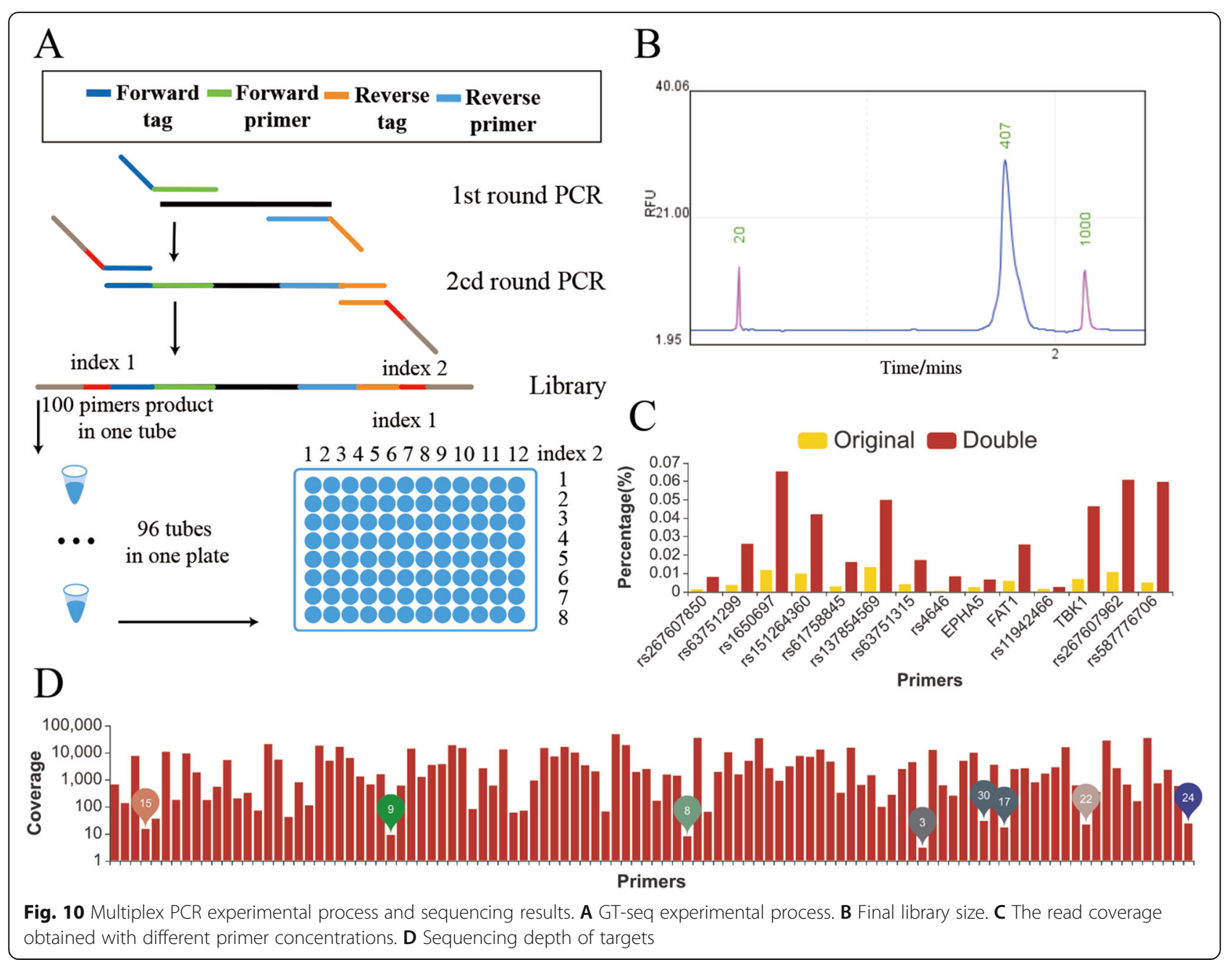

adjust their strategy to obtain the optimal multiplex primer clusters. The report displays a summary of the design, filtering, information clustering and redesign, and the detailed reasons for failure at any step. According to the report, the user is allowed to choose the generated primers or to change the strategy to generate a more specific primer set. With Ultiplex, we designed primers for 271 targets pooled in one reaction. The results showed that this primer set could achieve a $93 \%$ success rate. For genome mutation, this multiplex system stably detected $0.25 \%$ mutation rates. Providing user-friendly reports and web-based interface, Ultiplex will assist in biological applications and research involving genomic variants.

Table 4 Mutation rates of simulated samples of rs28934573 (C > T)

\begin{tabular}{|c|c|c|c|c|c|c|c|}
\hline Mixture Ratio & Theoretical value & Mean & Repeat 1 & Repeat 2 & Repeat 3 & Variance & CV \\
\hline HCT-15 & $50.00 \%$ & $50.64 \%$ & $51.04 \%$ & $49.52 \%$ & $51.37 \%$ & $0.99 \%$ & $1.95 \%$ \\
\hline $\mathrm{HaCaT}$ & $0.00 \%$ & $0.11 \%$ & $0.13 \%$ & $0.10 \%$ & $0.10 \%$ & $0.02 \%$ & $15.75 \%$ \\
\hline $50 \%$ HCT-15/HaCaT & $16.67 \%$ & $17.42 \%$ & $16.59 \%$ & $17.80 \%$ & $17.87 \%$ & $0.72 \%$ & $4.13 \%$ \\
\hline 25\% HCT-15/HaCaT & $10.00 \%$ & $11.04 \%$ & $11.86 \%$ & $10.89 \%$ & $10.37 \%$ & $0.76 \%$ & $6.85 \%$ \\
\hline 10\% HCT-15/HaCaT & $4.55 \%$ & $5.34 \%$ & $5.25 \%$ & $5.75 \%$ & $5.03 \%$ & $0.37 \%$ & $6.91 \%$ \\
\hline 5\% HCT-15/HaCaT & $2.38 \%$ & $2.82 \%$ & $2.50 \%$ & $2.48 \%$ & $3.47 \%$ & $0.57 \%$ & $20.09 \%$ \\
\hline 1\% HCT-15/HaCaT & $0.50 \%$ & $0.63 \%$ & $0.68 \%$ & $0.37 \%$ & $0.84 \%$ & $0.24 \%$ & $37.93 \%$ \\
\hline $0.5 \%$ HCT-15/HaCaT & $0.25 \%$ & $0.24 \%$ & $0.25 \%$ & $0.29 \%$ & $0.19 \%$ & $0.05 \%$ & $20.68 \%$ \\
\hline $0.1 \%$ HCT-15/HaCaT & $0.05 \%$ & $0.16 \%$ & $0.09 \%$ & $0.30 \%$ & $0.10 \%$ & $0.12 \%$ & $72.53 \%$ \\
\hline
\end{tabular}




\section{Availability and requirements \\ Project name: Ultiplex}

Project home page: http://ultiplex.igenebook.cn

Operating system(s): User interface: Platform independent; Server side: Linux

Programming language: Flask, SQLite, HTML, jQuery, and Python

Other requirements: Web browser (supporting JavaScript)

License: None for usage

Any restrictions to use by non-academics: Licenses for the number of designed primer pairs for each target will be limited to 20 to save computational resources.

\section{Abbreviations}

PCR: Polymerase chain reaction; HNPCC: Hereditary nonpolyposis colorectal cancer; MMR: Mismatch repair gene; BLAST: The Basic Local Alignment Search ToolTmMelting temperature; CPU: Central processing unit

\section{Supplementary Information}

The online version contains supplementary material available at https://doi. org/10.1186/s12864-021-08149-1.

Additional file 1: Figure S1. Amplification specificity and efficiency validation of single primer pairs. $S$ refers to the human genome DNA sample, and $\mathrm{N}$ refers to the water negative control. Primer pair IDs are listed in Table S2.

Additional file 2.

Additional file 3.

Additional file 4

Additional file 5 .

Additional file 6 .

\section{Acknowledgements}

Not applicable.

\section{Authors' contributions}

JY1 has drafted the manuscript and drafted the concept of primers design for multiple PCR. JY2 has drafted the work, designed the code of the software, and analyzed the interpretation of data. MZ, QX, and TZ have participated in the work of experimental verification. JZ designed the nextgeneration sequencing data processing pipeline and genome variants detection pipeline. ZQL has designed the conception of software and provided financial support. ZL has designed the experimental verification and provided financial support. All authors read and approved the final version of the manuscript.

\section{Funding}

This work was supported by the Guangdong Medical Research Fund [A2018096]. Jie Yuan's work was supported by Dongping talent plan of Foshan Fosun Chancheng Hospital. The funding body played no role in the design of the study and collection, analysis, and interpretation of data and in writing the manuscript.

\section{Availability of data and materials}

All data generated or analysed during this study are included in this published article and its Supplementary files, as well as in Genome Sequence Archive (https://ngdc.cncb.ac.cn/gsa-human/) under specific accession number (HRA001504). The Ultiplex multiplex primer design tool is available at http:// ultiplex.igenebook.cn.The guidance video for this website is available at https://www.youtube.com/watch?v=Fm4b8yWyEgM.

\section{Declarations}

Ethics approval and consent to participate

Not applicable

\section{Consent for publication}

Not Applicable

\section{Competing interests}

The authors declare that they have no competing interests.

\section{Author details}

${ }^{1}$ General Surgery Center, Zhujiang Hospital, Southern Medical University, 253 Industrial Boulevard, Guangzhou 510280, Guangdong Province, China.

${ }^{2}$ Department of General Surgery, Foshan Fosun Chancheng Hospital, Foshan 528010, China. ${ }^{3}$ Medical Department, Wuhan Igenebook Biotechnology co. Ltd, Floor 3, building 1, Zone B, Gaonong Biological Park, 888 Gaoxin Avenue, Wuhan 430014, Hubei Province, China. ${ }^{4}$ Zhuhai Interventional Medical Center, Zhuhai City People's Hospital, Zhuhai 519000, China. ${ }^{5}$ College of Landscape Architecture, Central South University of Forestry and Technology, Changsha 410004, China.

Received: 5 May 2021 Accepted: 4 November 2021

Published online: 18 November 2021

\section{References}

1. Genomes Project C, Auton A, Brooks LD, Durbin RM, Garrison EP, Kang HM, et al. A global reference for human genetic variation. Nature. 2015; 526(7571):68-74.

2. Hamosh A, Scott AF, Amberger J, Bocchini C, Valle D, McKusick VA. Online Mendelian Inheritance in Man (OMIM), a knowledgebase of human genes and genetic disorders. Nucleic Acids Res. 2002;30(1):52-5. https://doi.org/1 $0.1093 / \mathrm{nar} / 30.1 .52$

3. Bhattacharya P, McHugh TW. Lynch syndrome. Treasure Island: In: StatPearls; 2020

4. Wang S, Zhang S, Zeng Z, Ou Y, Han M, Guo J, et al. Association of MMR protein expression and MMR gene mutations in Chinese colorectal cancer patients. Ann Oncol. 2018;29:viii52. https://doi.org/10.1093/annonc/mdy2 69.164.

5. Caja F, Vodickova L, Kral J, Vymetalkova V, Naccarati A, Vodicka P. DNA mismatch repair gene variants in sporadic solid cancers. Int J Mol Sci. 2020; 21(15). https://doi.org/10.3390/ijms21155561.

6. Giolai M, Paajanen P, Verweij W, Percival-Alwyn L, Baker D, Witek K, et al. Targeted capture and sequencing of gene-sized DNA molecules. Biotechniques. 2016;61(6):315-22. https://doi.org/10.2144/000114484.

7. Henegariu O, Heerema NA, Dlouhy SR, Vance GH, Vogt PH. Multiplex PCR: critical parameters and step-by-step protocol. Biotechniques. 1997;23(3): 504-11. https://doi.org/10.2144/97233rr01.

8. Untergasser A, Cutcutache I, Koressaar T, Ye J, Faircloth BC, Remm M, et al. Primer3--new capabilities and interfaces. Nucleic Acids Res. 2012;40(15):e115. https://doi.org/10.1093/nar/gks596.

9. Ye J, Coulouris G, Zaretskaya I, Cutcutache I, Rozen S, Madden TL. PrimerBLAST: a tool to design target-specific primers for polymerase chain reaction. BMC Bioinformatics. 2012;13(1):134. https://doi.org/10.1186/1471-21 05-13-134.

10. Rychlik W. OLIGO 7 primer analysis software. Methods Mol Biol. 2007;402: 35-60. https://doi.org/10.1007/978-1-59745-528-2_2.

11. Graham KJ, Holland MJ. PrimerSelect: a transcriptome-wide oligonucleotide primer pair design program for kinetic RT-PCR-based transcript profiling. Methods Enzymol. 2005;395:544-53. https://doi.org/10.1016/S00766879(05)95028-3.

12. Singh VK, Mangalam AK, Dwivedi S, Naik S. Primer premier: program for design of degenerate primers from a protein sequence. Biotechniques. 1998;24(2):318-9. https://doi.org/10.2144/98242pf02.

13. Rachlin J, Ding C, Cantor C, Kasif S. MuPlex: multi-objective multiplex PCR assay design. Nucleic Acids Res. 2005;33(Web Server issue):W544-7.

14. Camacho C, Coulouris G, Avagyan V, Ma N, Papadopoulos J, Bealer K, et al. BLAST+: architecture and applications. BMC Bioinformatics. 2009;10(1):421. https://doi.org/10.1186/1471-2105-10-421.

15. Dale RK, Pedersen BS, Quinlan AR. Pybedtools: a flexible Python library for manipulating genomic datasets and annotations. Bioinformatics. 2011; 27(24):3423-4. https://doi.org/10.1093/bioinformatics/btr539. 
16. Chen S, Zhou Y, Chen Y, Gu J. fastp: an ultra-fast all-in-one FASTQ preprocessor. Bioinformatics. 2018;34(17):1884-90. https://doi.org/10.1093/ bioinformatics/bty560.

17. Langmead B, Salzberg SL. Fast gapped-read alignment with bowtie 2. Nat Methods. 2012;9(4):357-9. https://doi.org/10.1038/nmeth.1923.

18. Li H, Handsaker B, Wysoker A, Fennell T, Ruan J, Homer N, et al. The sequence alignment/map format and SAMtools. Bioinformatics. 2009;25(16): 2078-9. https://doi.org/10.1093/bioinformatics/btp352.

19. Danecek P, McCarthy SA. BCFtools/csq: haplotype-aware variant consequences. Bioinformatics. 2017;33(13):2037-9. https://doi.org/10.1093/ bioinformatics/btx 100 .

20. Untergasser A, Nijveen H, Rao X, Bisseling T, Geurts R, Leunissen JA. Primer3Plus, an enhanced web interface to Primer3. Nucleic Acids Res. 2007; 35(Web Server issue):W71-4.

21. Head SR, Komori HK, LaMere SA, Whisenant T, Van Nieuwerburgh F, Salomon DR, et al. Library construction for next-generation sequencing: overviews and challenges. Biotechniques. 2014;56(2):61-64, 66, 68, passim.

22. Campbell NR, Harmon SA, Narum SR. Genotyping-in-thousands by sequencing (GT-seq): a cost effective SNP genotyping method based on custom amplicon sequencing. Mol Ecol Resour. 2015;15(4):855-67. https:// doi.org/10.1111/1755-0998.12357.

23. You FM, Huo N, Gu YQ, Luo MC, Ma Y, Hane D, et al. BatchPrimer3: a high throughput web application for PCR and sequencing primer design. BMC Bioinformatics. 2008;9(1):253. https://doi.org/10.1186/1471-2105-9-253.

24. Francis F, Dumas MD, Wisser RJ. ThermoAlign: a genome-aware primer design tool for tiled amplicon resequencing. Sci Rep. 2017;7(1):44437. https://doi.org/10.1038/srep44437.

25. Wang Y. A multiplex PCR primer designing system for target sequencing. Shanghai: Donghua University; 2018.

26. Koressaar T, Remm M. Enhancements and modifications of primer design program Primer3. Bioinformatics. 2007;23(10):1289-91. https://doi.org/10.1 093/bioinformatics/btm091.

27. Hendling M, Pabinger S, Peters K, Wolff N, Conzemius R, Barisic I. Olizgo: an automated multiplex oligonucleotide design tool. Nucleic Acids Res. 2018; 46(W1):W252-6. https://doi.org/10.1093/nar/gky319.

\section{Publisher's Note}

Springer Nature remains neutral with regard to jurisdictional claims in published maps and institutional affiliations.

Ready to submit your research? Choose BMC and benefit from:

- fast, convenient online submission

- thorough peer review by experienced researchers in your field

- rapid publication on acceptance

- support for research data, including large and complex data types

- gold Open Access which fosters wider collaboration and increased citations

- maximum visibility for your research: over $100 \mathrm{M}$ website views per year

At $\mathrm{BMC}$, research is always in progress.

Learn more biomedcentral.com/submissions 